\title{
Existencia y multiplicidad de soluciones para problemas elípticos semilineales y cuasilineales
}

\author{
Jorge Cossio* \\ Escuela de Matemáticas, Universidad Nacional de Colombia Sede Medellín, Colombia
}

\section{Resumen}

En este artículo se presentan los resultados más importantes de mi trabajo de investigación en el estudio de la existencia, multiplicidad y propiedades cualitativas de las soluciones de problemas elípticos semilineales del tipo

$$
\left\{\begin{array}{rll}
\Delta u+f(u) & =0 & \text { en } \Omega \\
u & =0 & \text { en } \partial \Omega
\end{array}\right.
$$

y de problemas elípticos cuasilineales de la forma

$$
\left\{\begin{aligned}
\Delta_{p} u+f(u)=0 & \text { en } \Omega, \\
u=0 & \text { en } \partial \Omega,
\end{aligned}\right.
$$

donde $\Delta$ es el operador de Laplace, $\Delta_{p}$ es el $p$-laplaciano, $\Omega \subset \mathbb{R}^{N}(N \geq 2)$ es un dominio acotado en $\mathbb{R}^{N}$ con frontera suave y $f: \mathbb{R} \rightarrow \mathbb{R}$ es una función no lineal. Los teoremas que presentamos han sido obtenidos utilizando métodos variacionales, un principio de minimax demostrado en Castro, Cossio \& Neuberger (1997), teoría de grado, teoría de Morse y teoría de bifurcación. Además se formulan una serie de preguntas abiertas relacionadas con los problemas (1) y (2), que esperamos sean de interés para los analistas no lineales.

Palabras claves: Ecuaciones elípticas semilineales, ecuaciones elípticas cuasilineales, métodos variacionales, reducción de Lyapunov-Schmidt, teoría de grado, teoría de Morse, teoría de bifurcación.

Existence and multiplicity of solutions to semilinear and quasilinear elliptic problems

\section{Abstract}

In this paper I present some results of my research studying existence, multiplicity, and qualitative properties of solutions to semilinear elliptic problems of the type

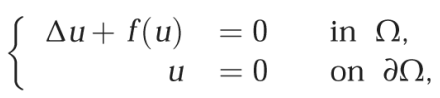

and to quasilinear elliptic problems of the type

$$
\left\{\begin{aligned}
\Delta_{p} u+f(u)=0 & & \text { in } \Omega, \\
u=0 & & \text { on } \partial \Omega,
\end{aligned}\right.
$$

where $\Delta$ is the Laplace operator, $\Delta_{p}$ is the $p$-Laplacian, $\Omega \subset \mathbb{R}^{N}(N \geq 2)$ is a bounded domain in $\mathbb{R}^{N}$ with smooth boundary, and $f: \mathbb{R} \rightarrow \mathbb{R}$ is a nonlinear function. Our theorems were obtained by using variational methods, a minmax principle developed in Castro, Cossio \& Neuberger (1997), Leray-Schauder degree, Morse theory, and bifurcation theory. Besides statement of principal results, we present some open problems which seem of interest for nonlinear analysts.

Key words: Semilinear elliptic equations, quasilinear elliptic equations, variational methods, Lyapunov-Schmidt reduction, Leray-Schauder degree, Morse theory, bifurcation theory.

*Correspondencia: Jorge Cossio: jcossio@unal.edu.co. Recibido enero 2015; aceptado abril 2015. 


\section{Introducción}

En este trabajo se estudia inicialmente la existencia de soluciones para problemas elípticos semilineales del tipo

$$
\left\{\begin{aligned}
\Delta u+f(u)=0 & \text { en } \Omega, \\
u=0 & \text { en } \partial \Omega,
\end{aligned}\right.
$$

donde $\Delta$ es el operador de Laplace, $\Omega \subset \mathbb{R}^{N}(N \geq 2)$ es un dominio acotado en $\mathbb{R}^{N}$ con frontera suave y $f: \mathbb{R} \rightarrow \mathbb{R}$ es una función no lineal.

Los problemas elípticos semilineales modelan una gran variedad de problemas que aparecen en distintas áreas del conocimiento, tales como geometría, física, ingeniería, astrofísica y biología. A continuación se presentan algunos ejemplos donde aparecen ecuaciones elípticas semilineales:

(i) Las soluciones estacionarias de ecuaciones del calor no lineales del tipo

$$
u_{t}-\Delta u=f(u)
$$

(ii) Las soluciones estacionarias de la ecuación de Schrödinger no lineal

$$
i u_{t}+\Delta u=k|u|^{p} u
$$

(iii) La ecuación Sine-Gordon

$$
-\Delta u+\sin u=0,
$$

que aparece en geometría cuando se estudian superficies de curvatura constante negativa.

(iv) La ecuación

$$
\Delta u+4 \pi\left(2 u+u^{2}\right)^{\frac{3}{2}}=0,
$$

que aparece en astrofísica en el estudio de problemas estelares.

(v) Las ecuaciones de campo escalar que se estudian en física clásica y cuántica tienen la forma

$$
-\Delta u=f(u)
$$

Cuando se estudia el problema (5) aparecen varias preguntas importantes:

(i) ¿Existen soluciones?

(ii) Si existe solución, ¿es ésta única o existen múltiples soluciones?

(iii) Si existen soluciones, ¿qué propiedades cualitativas tienen dichas soluciones?
Consideremos el problema de valores propios para el laplaciano:

$$
\left\{\begin{aligned}
\Delta u+\lambda u=0 & \text { en } \Omega, \\
u=0 & \text { en } \partial \Omega .
\end{aligned}\right.
$$

El valor del parámetro $\lambda \in \mathbb{R}$ para el cual (6) tiene una solución $u \neq 0$ se llama un valor propio de (6) y la correspondiente función $u$ se llama una función propia asociada con $\lambda$. Denotamos por $0<\lambda_{1}<\lambda_{2} \leq \cdots \leq \lambda_{k} \leq \cdots$ la sucesión de valores propios de (6).

Es bien conocido que el conjunto de valores propios forma una sucesión creciente que tiende a infinito y que la existencia de soluciones del problema (5) depende de la posición de la derivada de la no linealidad $f$ con respecto a los valores propios.

En el estudio del problema (5) se distinguen dos casos:

(I) Problemas asintóticamente lineales, en los cuales

$$
f^{\prime}(\infty):=\lim _{|t| \rightarrow \infty} f^{\prime}(t) \in \mathbb{R} .
$$

En este caso consideramos dos subcasos:

(Ia) Resonancia. Decimos que (5) es un problema resonante si

$$
f^{\prime}(\infty)=\lambda_{k}, \quad \text { para algún } k \in \mathbb{N} .
$$

(Ib) No resonancia. Decimos que (5) es un problema no resonante si

$$
f^{\prime}(\infty) \neq \lambda_{k}, \quad \text { para todo } k \in \mathbb{N} .
$$

(II) Problemas superlineales, en los cuales

$$
f^{\prime}(\infty)=\lim _{|t| \rightarrow \infty} f^{\prime}(t)=\infty .
$$

Los problemas elípticos semilineales pueden ser estudiados con una amplia variedad de métodos y técnicas que han sido desarrollados en muchos centros de investigación alrededor del mundo y que han dedicado un gran esfuerzo al entendimiento de este tipo de problemas no lineales.

En la Sección 2 de este trabajo presentamos algunos de los resultados más importantes que hemos obtenido estudiando el problema (5) tanto en el caso asintóticamente lineal como en el caso superlineal. Para este estudio hemos utilizado varios métodos. En primer lugar hemos usado los métodos variacionales, en los cuales la existencia de soluciones del problema (5) está relacionada con la existencia de puntos críticos de un funcional asociado con la ecuación elíptica semilineal. Uno de los métodos específicos para encontrar puntos críticos es el bien conocido Teorema del Paso de la Montaña, publicado por 
Ambrosetti \& Rabinowitz (1973). Otro resultado importante para encontrar puntos críticos es el método de reducción de Lyapunov-Schmidt, el cual tuvo sus orígenes en las investigaciones de los profesores Lazer, Landesman y Meyers (véase Landesman, Lazer \& Meyers (1975)) y Castro y Lazer (véase Castro \& Lazer (1976)), realizadas en los años setenta del siglo pasado, y que permite reducir el estudio de los puntos críticos del funcional asociado, el cual está generalmente definido en un espacio de dimensión infinita, al estudio de los puntos críticos de un funcional definido en un subespacio, generalmente de dimensión finita. Además de estos dos métodos, en la Sección 2 de este trabajo se utilizan un principio de minimax, desarrollado en Castro, Cossio \& Neuberger (1997), que permite obtener soluciones que cambian de signo para problemas superlineales y la teoría de Morse, desarrollada inicialmente por Morse en Morse (1925) y Morse (1934) y posteriormente por diferentes investigadores, entre los que destacamos los trabajos de R. Palais y $\mathbf{S}$. Smale en Palais \& Smale (1964) y K. C. Chang en Chang (1993).

Otra herramienta importante que hemos usado en este estudio es la teoría de bifurcación. Bifurcación significa cambios en la estructura del conjunto de soluciones de una ecuación funcional cuando los parámetros que intervienen en la ecuación varían. Las ideas fundamentales de la teoría de bifurcación que hemos utilizado están basadas en los trabajos de M. Crandall y P. Rabinowitz (1971) , en lo concerniente a la existencia de ramas locales de soluciones, y de P. Rabinowitz (1971), en lo relacionado con la existencia de ramas globales de soluciones; a partir de estos trabajos se han hecho contribuciones muy importantes en esta teoría que han permitido su desarrollo, destacamos los trabajos de Ambrosetti \& Malchiodi (2007) y Ambrosetti \& Prodi (1993).

Utilizando el teorema del paso de la montaña, el método de reducción de Lyapunov-Schmidt, el principio de minimax de Castro, Cossio \& Neuberger (1997), la teoría de Morse, la teoría de grado y la teoría de bifurcación hemos conseguido varios resultados en el estudio de los problemas elípticos semilineales (véanse Cossio \& Vélez (2003), Cossio \& Herrón (2004), Cossio, Herrón \& Vélez (2013), Cossio, Herrón \& Vélez (2009), Castro, Cossio \& Vélez (2013), Castro \& Cossio (2006)). Estos teoremas serán presentados en la Sección 2 de este trabajo. Debo mencionar que acá sólo se presentan los resultados de mi trabajo de investigación obtenidos a partir del año 2003, para mis trabajos anteriores refiero al lector al artículo Cossio (2004).

La existencia de soluciones para problemas elípticos semilineales del tipo (5) y sus propiedades cualitati- vas han sido estudiadas por muchos autores, entre ellos mencionamos los trabajos Ambrosetti \& Hesse (1980), Ambrosetti \& Malchiodi (2007), Ambrosetti \& Prodi (1993), Ambrosetti \& Rabinowitz (1973), Bartsch, Chang \& Wang (2000), Bartsch \& Wang (1996), Bartsch \& Weth (2003), Castro (1981), Castro \& Cossio (1994), Castro \& Cossio (1993), Castro, Cossio \& Neuberger (1997A), Castro, Cossio \& Neuberger (1998), Castro \& Lazer (1976), Castro \& Lazer (1979), Chang (1981), Chang (1993), Chang, Li \& Liu (1994), Cossio (1996), Cossio (2004), Dancer (1976), Genoud (2011), Liu (2007), Lazer \& Solimini (1988), Ma, Xu \& Han (2011), Rabinowitz (1971), Rabinowitz (1973), Rabinowitz (1985), Rabinowitz (1986), Rabinowitz, Su \& Wang (2007), Wang (1991).

Hagamos

$$
\Delta_{p} u:=\operatorname{div}\left(|\nabla u|^{p-2} \nabla u\right), \quad p>1 .
$$

E1 operador

$$
u \longrightarrow \Delta_{p} u
$$

se llama el $p$-laplaciano. Obviamente, $\Delta_{2}=\Delta$ es el operador de Laplace. Observamos que para $p \neq 2$ el $p$ laplaciano es un operador $(p-1)$-homogéneo no lineal. En la segunda parte de este trabajo se estudian los problemas elípticos cuasilineales del tipo

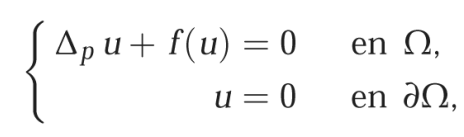

donde $\Omega$ es un conjunto abierto acotado en $\mathbb{R}^{N}, N \geq 2$, y $f: \mathbb{R} \rightarrow \mathbb{R}$ es una función no lineal.

Un aspecto interesante desde el punto de vista matemático es la gran diferencia que existe entre los problemas elípticos cuasilineales $(p \neq 2)$ y los problemas elípticos semilineales $(p=2)$, que como se ha demostrado en estos últimos años requieren gran tiempo para su entendimiento. El estudio del p-laplaciano es un reto y su estudio conducirá al desarrollo de nuevas técnicas y métodos que permitirán el desarrollo del análisis no lineal.

Es bien conocido que ciertos modelos matemáticos no lineales conducen a ecuaciones diferenciales que contienen el $p$-laplaciano. Por ejemplo, cuando se estudia el comportamiento de un fluido compresible en un medio poroso homogéneo e isotrópico, se llega a una ecuación parabólica cuasilineal del tipo

$$
u_{t}=\Delta_{p} u+f(u)
$$

que en el caso estacionario se reduce a

$$
\Delta_{p} u+f(u)=0 .
$$


Consideremos el problema de valores propios para el $p$ laplaciano:

$$
\left\{\begin{array}{rlrl}
\Delta_{p} u+\lambda|u|^{p-2} u=0 & & \text { en } \Omega, \\
u & =0 & & \text { en } \partial \Omega .
\end{array}\right.
$$

El valor del parámetro $\lambda \in \mathbb{R}$ para el cual (8) tiene una solución $u \neq 0$ se llama un valor propio de (8) y la correspondiente función $u$ se llama una función propia asociada con $\lambda$. En dimensión uno la estructura de valores propios y de vectores propios está bien estudiada, en este caso el conjunto de valores propios forma una sucesión creciente

$$
0<\lambda_{1}<\lambda_{2}<\cdots<\lambda_{n}<\cdots
$$

que tiende a infinito y cada $\lambda_{n}$ es simple en el sentido que hay exactamente una función propia $u_{n}$ asociada con $\lambda_{n}$ normalizada por $u_{n}(0)=1$. En dimensiones más altas la situación es diferente y más complicada. Se sabe que el primer valor propio $\lambda_{1}$ es simple y aislado y la correspondiente función propia $\varphi_{1}$ puede ser tomada positiva Anane (1987). Es también conocido que existe un mínimo valor propio $\lambda_{2}$, próximo a $\lambda_{1}$, llamado el segundo valor propio. Éste tiene una caracterización de tipo minimax y cualquier función propia asociada con él cambia de signo exactamente una vez en $\Omega$ (Anane \& Tsouli (1996), Drábek \& Robinson (1999)). Usando fórmulas de minimax es posible construir una sucesión $\lambda_{n}$ de valores propios, llamados valores propios variacionales, que se aproxima a infinito. En el caso $p=2$ estos son los únicos valores propios de (8). El problema de la existencia de otros valores propios de (8) distintos de los valores propios variacionales permanece abierto si $p \neq 2$, como también cuántos valores propios no variacionales hay y si el segundo valor propio $\lambda_{2}$ es aislado o no.

Recordamos que las soluciones radiales del problema (7), en el caso en que $\Omega=\left\{x \in \mathbb{R}^{N}:\|x\|<1\right\}$ es la bola unitaria en $\mathbb{R}^{N}$, corresponden a soluciones del problema

$$
\left\{\begin{aligned}
\left(r^{N-1}\left(\left|u^{\prime}\right|^{p-2} u^{\prime}\right)\right)^{\prime}+r^{N-1} f(u(r)) & =0, \quad r \in(0,1) \\
u^{\prime}(0)=0 & =u(1) .
\end{aligned}\right.
$$

Sea $\left\{\lambda_{k}(p)\right\}_{k \in \mathbb{N}}$ la sucesión de valores propios radiales del problema de valores propios

$$
\left\{\begin{aligned}
\left(r^{N-1}\left(\left.\left|u^{\prime}\right|\right|^{p-2} u^{\prime}\right)\right)^{\prime}+\lambda r^{N-1}\left|u^{\prime}\right|^{p-2} u^{\prime} & =0, r \in(0,1) \\
u^{\prime}(0)=0 & =u(1) .
\end{aligned}\right.
$$

Es conocido que la sucesión de valores propios radiales $\left\{\lambda_{k}(p)\right\}_{k \in \mathbb{N}}$ es creciente, tiende a infinito y cada valor propio es simple.
Hagamos

$$
f_{p}^{\prime}(0):=\lim _{u \rightarrow 0} \frac{f(u)}{|u|^{p-2} u}
$$

$\mathrm{y}$

$$
f_{p}^{\prime}(\infty):=\lim _{|u| \rightarrow \infty} \frac{f(u)}{|u|^{p-2} u} .
$$

Llamaremos $f_{p}^{\prime}(0)$ la $p$-derivada en 0 y $f_{p}^{\prime}(\infty)$ la $p$-derivada en $\infty$.

Decimos que la no linealidad es:

- (a) p-asintóticamente lineal en el origen si $f_{p}^{\prime}(0) \in \mathbb{R}$

- (b) p-asintóticamente lineal en el infinito si $f_{p}^{\prime}(\infty) \in \mathbb{R}$

- (c) p-superlineal en el origen si $f_{p}^{\prime}(0)=\infty \mathrm{y}$

- (d) p-superlineal en el infinito si $f_{p}^{\prime}(\infty)=\infty$.

En la Sección 3 de este trabajo, presentamos algunos resultados relacionados con la existencia de múltiples soluciones radiales para el problema (7) cuando la no linealidad tiene un cero positivo y el rango de la $p$-derivada de la no linealidad $f$ incluye al menos los primeros $j$ valores propios radiales del operador $p$-laplaciano o cuando la no linealidad es $p$-asintóticamente superlineal en el origen (véanse los Teoremas 3.1, 3.2, 3.3). Para demostrar estos resultados las técnicas que utilizamos son teoría de bifurcación y el "shooting method" (método del disparo).

Además, demostramos la existencia de múltiples soluciones para el problema (7), cuando la $p$-derivada en cero y la $p$-derivada en infinito son mayores que el primer valor propio del $p$-laplaciano. Este resultado extiende un trabajo de J. Cossio, S. Herrón C. Vélez obtenido para el caso semilineal (véase Cossio, Herrón \& Vélez (2013)). La demostración utiliza el teorema de bifurcación global, bifurcación de infinito y una versión no lineal del principio del máximo. Además se obtienen algunas propiedades cualitativas de las soluciones.

Existen muchos resultados relacionados con la existencia de soluciones para el problema (7) tanto en el caso radial como en el no radial. Al lector interesado le sugerimos las referencias Bognár \& Drábek (2005), Capietto, Dambrosio \& Zanolin ( 2001), Cossio \& Herrón (2008), Cossio, Herrón \& Vélez (2011), Del Pino \& Manásevich (1991), Drábek \& Robinson (1999), García-Melián \& Sabina de Lis (2002), El Hachimi \& De Thelin (1996), Iaia (1995), Garcia-Huidrobo, Manásevich \& Schmitt (1999), O'Regan \& Wang (2008).

Además de la presentación en este trabajo de los resultados más importantes obtenidos por el autor en colaboración con otros investigadores en el área, en la Sección 4 
presentamos una serie de preguntas abiertas relacionadas con los problemas elípticos semilineales y cuasilineales, que esperamos sean de interés para los analistas no lineales y contribuyan a un mejor entendimiento de este tipo de problemas.

\section{Problemas elípticos semilineales}

En esta sección se presentan los resultados más importantes de mi trabajo de investigación en el estudio de la existencia, multiplicidad, y propiedades cualitativas de las soluciones de problemas elípticos semilineales de la forma

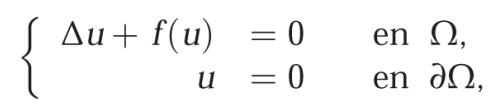

donde $\Omega \subset \mathbb{R}^{N}, N>2$, es un dominio acotado en $\mathbb{R}^{N}$ con frontera suave y $f: \mathbb{R} \rightarrow \mathbb{R}$ es una función no lineal tal que $f(0)=0$.

Cuando se utilizan los métodos variacionales para estudiar el problema (10) (véase Cossio (2004) y las referencias citadas allí), las soluciones de (10) son los puntos críticos del funcional $J: H_{0}^{1}(\Omega) \rightarrow \mathbb{R}$ definido por

$$
J(u)=\int_{\Omega}\left(\frac{1}{2}|\nabla u|^{2}-F(u)\right) d x,
$$

donde $F(\xi)=\int_{0}^{\xi} f(s) d s$.

En el caso en que la no linealidad sea asintóticamente lineal, el funcional $J \in C^{2}$ (véase Rabinowitz (1973)) y, además,

$$
\begin{array}{r}
D J(u) v=\langle\nabla J(u), v\rangle=\int_{\Omega}(\nabla u \cdot \nabla v-f(u) v) d x, \\
\forall u, v \in H_{0}^{1}(\Omega), \\
\left\langle D^{2} J(u) v, w\right\rangle=\int_{\Omega}\left(\nabla v \cdot \nabla w-f^{\prime}(u) v W\right) d x \\
\forall u, v, w \in H_{0}^{1}(\Omega) .
\end{array}
$$

Si $J \in C^{2}$ y $u_{0}$ es un punto crítico de $J$ definimos el índice de Morse de $J$ en $u_{0}$ como sigue: si existe un entero no negativo $m$ tal que existe un subespacio $m$-dimensional de $H$ en el cual $D^{2} J\left(u_{0}\right)$ está definido negativamente y $m$ es maximal con respecto a esta propiedad, decimos que $m$ es el índice de Morse de $J$ en $u_{0}$ y se denota por $m\left(J, u_{0}\right)$ o por $m\left(u_{0}\right)$ cuando no hay lugar a confusión. Si tal $m$ no existe decimos que índice de Morse de $J$ en $u_{0}$ es infinito. De manera similar se define el índice de Morse aumentado $m_{a}\left(u_{0}\right)$ de $J$ en $u_{0}$, cambiando la expresión "definido negativamente" por "definido no positivamente". Un punto crítico $u_{0}$ de $J$ se dice no degenerado si $D^{2} J\left(u_{0}\right)$ es invertible.

En Cossio \& Vélez (2003), en colaboración con C. Vélez, estudiamos el problema (10) en el caso en que la no linealidad $f$ es asintóticamente lineal y demostramos que el problema (10) tiene por lo menos tres soluciones no triviales. Utilizamos argumentos del tipo de paso de la montaña Ambrosetti \& Rabinowitz (1973) para demostrar la existencia de dos soluciones de un signo y la teoría de grado de Leray-Schauder para probar la existencia de otra solución que cambia de signo.

Teorema 2.1. (Cossio \& Vélez (2003)) Si $f^{\prime}(0)<\lambda_{1}$, $f^{\prime}(\infty) \in\left(\lambda_{k}, \lambda_{k+1}\right)$ con $k$ un entero par, $k \geq 2$, entonces (10) tiene por lo menos tres soluciones no triviales, una es positiva, otra es negativa y la tercera cambia de signo.

En colaboración con S. Herrón, en Cossio \& Herrón (2004), demostramos la existencia de por lo menos tres soluciones para el problema (10) sin la restricción sobre la paridad de $k$ que se tiene en el Teorema 2.1, pero incluyendo una condición de no degeneramiento de los puntos críticos del funcional $J$. En Cossio \& Herrón (2004) se obtiene, además, información cualitativa sobre el índice de Morse de las soluciones. Para la prueba de este resultado usamos el teorema del paso de la montaña, el teorema de punto de silla de P. Rabinowitz (véase Rabinowitz (1986)) y argumentos del índice de Morse desarrollados por A. Lazer y S. Solimini (véase Lazer \& Solimini (1988)).

Teorema 2.2. (Cossio \& Herrón (2004)) Si $f^{\prime}(0)<\lambda_{1}$, $f^{\prime}(\infty) \in\left(\lambda_{k}, \lambda_{k+1}\right)$ con $k \geq 2, y$ todos los puntos críticos del funcional $J$ son no degenerados entonces (10) tiene por 10 menos tres soluciones no triviales: $u_{1}>0$ en $\Omega, u_{2}<0$ en $\Omega$ y $u_{3}$. Las soluciones de un signo tienen índice de Morse menor o igual a 1 y $u_{3}$ tiene índice de Morse mayor o igual a 2.

Observamos que K. C. Chang (1981) demostró también la existencia de al menos tres soluciones no triviales del problema (10), cuando la no linealidad satisface $f^{\prime}(0)>\lambda_{2}$ ó $f^{\prime}(0)=\lambda_{2}$ con $f(s) / s \geq f^{\prime}(0)$ en una vecindad $U$ de $s=0$. En su prueba Chang utilizó la teoría de Morse.

J. Cossio, S. Herrón y C. Vélez (véase Cossio, Herón \& Vélez (2009)), utilizando la teoría de grado de Leray-Schauder y resultados de A. Lazer y S. Solimini (véase Lazer \& Solimini (1988)), demostraron que el problema (10) tiene por lo menos tres soluciones no triviales cuando el rango de la derivada de la no linealidad incluye al menos los primeros $k$ valores propios de $-\Delta$, sin ninguna restricción sobre el no degeneramiento de los puntos críticos del funcional $J$ ni sobre la paridad de $k$. Este resultado da una respuesta parcial a una conjetura formulada en Cossio \& Herrón (2004). 
Observamos que si se tienen las hipótesis $f^{\prime}(0)<\lambda_{1}$ y $f^{\prime}(\infty) \in\left(\lambda_{k}, \lambda_{k+1}\right)$ con $k \geq 2$ entonces, via el teorema del paso de la montaña, se prueba que existe una solución positiva $u_{+}$y una solución negativa $u_{-}$de (10).

Definamos

$$
v:=\max \left\{\operatorname{dim} \operatorname{ker} D^{2} J\left(\boldsymbol{u}_{+}\right), \operatorname{dim} \operatorname{ker} D^{2} J\left(u_{-}\right)\right\} .
$$

Teorema 2.3. (Cossio, Herrón \& Vélez (2009)) Supongamos que $f^{\prime}(0)<\lambda_{1}$ y $f^{\prime}(\infty) \in\left(\lambda_{k}, \lambda_{k+1}\right)$ con $k \geq 2$. Si $v \leq k-2$ entonces el problema (10) tiene al menos tres soluciones no triviales.

Regresando al caso no degenerado, J. Cossio, S. Herrón y C. Vélez probaron la existencia de por lo menos cuatro soluciones no triviales de (10) combinando un resultado de A. Lazer y S. Solimini (véase Lazer \& Solimini (1988)) con un argumento de conteo de grado de LeraySchauder. El enunciado preciso es el siguiente:

Teorema 2.4. (Cossio, Herrón \& Vélez (2009)) Si $f^{\prime}(0)<$ $\lambda_{1}, f^{\prime}(\infty) \in\left(\lambda_{k}, \lambda_{k+1}\right)$ con $k \geq 2$ y todos los puntos críticos de J son no degenerados entonces el problema (10) tiene al menos cuatro soluciones: $u_{+}>0$ en $\Omega, u_{-}<0$ en $\Omega$, û $y$ $u_{4}$. Las soluciones de un signo tienen índice de Morse uno y $\hat{u}$ tiene indice de Morse $k$.

Para $c \in \mathbb{R}$ denotemos por $J^{c}$ el conjunto

$$
J^{c}:=\left\{u \in H_{0}^{1}(\Omega) \mid J(u) \leq c\right\} .
$$

Si $J \in C^{1}$ y $u_{0}$ es un punto crítico aislado de $J$, definimos los grupos críticos de $J$ en $u_{0}$ de la siguiente manera

$$
C_{q}\left(J, u_{0}\right):=H_{q}\left(J^{c} \cap U, J^{c} \cap U \backslash\left\{u_{0}\right\}\right),
$$

donde $U$ es una vecindad de $u_{0}$ que no contiene otro punto crítico de $J, J\left(u_{0}\right)=c$ y $H_{q}(X, Y)$ denota el $q$-ésimo grupo de homología singular relativo del par topológico $(X, Y)$, tomando $\mathbb{R}$ como los coeficientes del grupo.

A. Castro, J. Cossio y C. Vélez (véase Castro, Cossio \& Vélez (2013)) estudiaron el problema (10) en el caso asintóticamente lineal no resonante, sin ninguna restricción sobre el no degeneramiento de los puntos críticos del funcional $J$ ni sobre la paridad de $k$, utilizando Lazer \& Solimini (1988) demostraron el siguiente resultado.

Teorema 2.5. (?) Supongamos que $f$ satisface $f^{\prime}(\infty) \in$ $\left(\lambda_{k}, \lambda_{k+1}\right)$ con $k \geq 2$. Entonces:

(a) Existe una solución $u_{0}$ de (10) tal que $C_{k}\left(J, u_{0}\right) \neq\{0\}$. En particular,

$$
m\left(u_{0}\right) \leq k \leq m_{a}\left(u_{0}\right) .
$$

(b) Si $f^{\prime}(0)<\lambda_{1}$ entonces (10) tiene al menos tres soluciones no triviales $u_{+}, u_{-} y u_{0}$. Además, $u_{+}>0$ en $\Omega$, $u_{-}<0$ en $\Omega, y C_{k}\left(J, u_{0}\right) \neq\{0\}$.

Después de haber probado el resultado anterior descubrimos que un resultado más general fue demostrado por Chang, Li and Liu usando argumentos similares (véase Chang, Li \& Liu (1994)).

En un artículo escrito en colaboración con los profesores A. Castro y J.M. Neuberger (véase Castro, Cossio \& Neuberger (1997)), demostramos un principio de minimax que nos permitió establecer condiciones suficientes que garantizan la existencia de soluciones que cambian de signo exactamente una vez para problemas de Dirichlet del tipo (10), cuando $f$ es superlineal y subcrítica. Recordamos que $f$ es subcrítica si

$$
|f(s)| \leq c_{1}|s|^{p}+c_{2}, \quad \text { con } \quad 1 \leq p<\frac{N+2}{N-2} .
$$

Teorema 2.6. (Castro, Cossio \& Neuberger (1997)) Si $f^{\prime}(0)<\lambda_{1}$ y $f$ es superlineal y subcrítica entonces (10) tiene al menos tres soluciones no triviales: $w_{1}>0$ en $\Omega, w_{2}<0$ en $\Omega$ y $w_{3}$. La función $w_{3}$ cambia de signo exactamente una vez en $\Omega$, es decir, $\left(w_{3}\right)^{-1}(\mathbb{R}-\{0\})$ tiene exactamente dos componentes conexas. Si son no degeneradas, las soluciones de un signo tienen índice de Morse 1 y la solución que cambia de signo tiene índice de Morse 2.

Este resultado es el primero en establecer la existencia de una solución del problema (10) que cambia de signo exactamente una vez.

Observamos que utilizando el principio de minimax mencionado arriba para problemas superlineales y los resultados de teoría de grado de A. Castro y J. Cossio (véase Castro \& Cossio (1994)), logramos demostar la existencia de soluciones que cambian de signo para problemas de Dirichlet asintóticamente lineales (véase Castro, Cossio \& Neuberger (1998)).

A. Castro y J. Cossio estudiaron el problema superlineal (véase Castro \& Cossio (2006))

$$
\left\{\begin{aligned}
\Delta u+|u|^{\alpha} u=0 & \text { en } B_{1}(0) \subset \mathbb{R}^{n}, \\
u=0 & \text { en } \partial B_{1}(0),
\end{aligned}\right.
$$

donde $B_{1}(0)$ es la bola unitaria con centro en el origen, $0<\alpha<\frac{4}{n-2}$ si $n>2, \alpha<\infty$ si $n=2$.

Si $u$ es una solución radial de (13) entonces $u$ satisface

$$
\left\{\begin{aligned}
u^{\prime \prime}+\frac{n-1}{r} u^{\prime}+|u|^{\alpha} u & =0 \quad(0<r \leq 1), \\
u(1)=u^{\prime}(0) & =0 .
\end{aligned}\right.
$$

Del Teorema 2 de Yanagida (1996) se sigue que para cada entero positivo $k$, (14) tiene exactamente una solución con $k$ ceros en $[0,1]$ y $u(0)>0$. 
En el caso $n=\alpha=2$, con ayuda de software, en Castro \& Cossio (2006) logramos construir una solución radial de (13) que cambia de signo exactamente una vez.

Nuestra construcción está basada en el estudio del problema de valor inicial

$$
\left\{\begin{array}{l}
v^{\prime \prime}+\frac{1}{r} v^{\prime}+v^{3}=0 \quad(r>0), \\
v(0)=1, v^{\prime}(0)=0 .
\end{array}\right.
$$

De este estudio concluimos el siguiente resultado.

Teorema 2.7. (Castro \& Cossio (2006)) Si $\alpha$ y $\tau$ son el primer y segundo segundo cero de la solución $v$ al problema (15) entonces

$$
\left\{\begin{aligned}
3.6115 & \leq \alpha \leq 3.6415, \\
-0.231514 & \leq v^{\prime}(\alpha) \leq-0.198982, \\
\tau & \geq 9.47, \\
\alpha^{2}\left(v^{\prime}(\alpha)\right)^{2} & \leq \int_{\alpha}^{\tau} s v^{4}(s) d s .
\end{aligned}\right.
$$

Combinando los estimativos del Teorema 2.7, la identidad de Pohozaev y la caracterización variacional de la solución de mínima energía del problema superlineal (13) que cambia de signo, dada en Castro, Cossio \& Neuberger (1997), concluimos que tal solución no puede ser radial (véanse también los resultados Aftalion \& Pacella (2004) y Bartsch, Weth \& Willem (2005)).

Teorema 2.8. (Castro \& Cossio (2006)) El problema

$$
\left\{\begin{aligned}
\Delta u+u^{3}=0 & \text { en } B_{1}(0) \subset \mathbb{R}^{2}, \\
u=0 & \text { en } \partial B_{1}(0),
\end{aligned}\right.
$$

tiene una solución que es no radial y cambia de signo exactamente una vez en $B_{1}(0)$.

Regresemos ahora al estudio del problema (10) en el caso asintóticamente lineal. En Castro, Cossio \& Vélez (2013) continuamos estudiando la existencia de soluciones que cambian de signo para problemas elípticos semilineales. Utilizando resultados clásicos de la teoría de ecuaciones diferenciales parciales de tipo elíptico, demostramos un estimativo a priori para las soluciones de un signo de (10).

Teorema 2.9. (Castro, Cossio \& Vélez (2013)) Dados $\epsilon>0, A>0$, y $D>0$ existe una constante positiva $B:=B(\epsilon, A, D, \Omega, N)$ tal que si $f$ satisface

(E1) $\left|f^{\prime}(t)\right| \leq D$ para todo $t \in \mathbb{R}$,

(E2) $f^{\prime}(t) \geq \lambda_{1}+\epsilon$, para todo $|t|>A$,

y $u$ es una solución positiva o negativa de (10) entonces $u$ satisface

$$
\|u\|_{L^{\infty}(\Omega)} \leq B
$$

Combinando las estimativas a priori del teorema anterior y la existencia de soluciones con índice de Morse aumentado del Teorema 2.5 demostramos los siguientes resultados para problemas asintóticamente lineales no resonantes.

Teorema 2.10. (Castro, Cossio \& Vélez (2013)) Supongamos que $f$ satisface $f^{\prime}(\infty) \in\left(\lambda_{k}, \lambda_{k+1}\right)$ con $k \geq 2$. Sean $\epsilon>0, A>0, D>0$, y $B>0$ como en el Teorema 2.9. Si

$$
f^{\prime}(t)<\lambda_{k} \quad \forall t \in[-B, B]
$$

entonces existe al menos una solución $u_{*}$ de (10) que cambia de signo y tal que

$$
\left\|u_{*}\right\|_{L^{\infty}(\Omega)}>B
$$

Teorema 2.11. (Castro, Cossio \& Vélez (2013)) Sea $f$ : $\mathbb{R} \rightarrow \mathbb{R}$ una función que satisface las siguientes condiciones:

(i) $f^{\prime}(\infty) \in\left(\lambda_{k}, \lambda_{k+1}\right)$ para algún $k \geq 2$,

(ii) Existe $\gamma>0$ tal que $f^{\prime}(t) \leq \gamma<\lambda_{k+1}$ para todo $t \in \mathbb{R}$,

(iii) $f^{\prime}(0)<\lambda_{1}$.

Sean $\epsilon>0, A>0, D>0$, y $B>0$ como en el Teorema 2.9. Si

$$
f^{\prime}(t)<\lambda_{k} \quad \forall t \in[-B, B]
$$

entonces existen al menos dos soluciones $u_{*} y v_{*}$ de (10) que cambian de signo. Además, una de ellas, digamos $u_{*}$ satisface

$$
\left\|u_{*}\right\|_{L^{\infty}(\Omega)}>B .
$$

Queremos mencionar que en Bartsch, Chang \& Wang (2000) se estudian problemas similares y se demuestra también la existencia de dos soluciones que cambian de signo.

En el caso asintóticamente lineal resonante tenemos el siguiente resultado.

Teorema 2.12. (Castro, Cossio \& Vélez (2013))Sea $f$ : $\mathbb{R} \rightarrow \mathbb{R}$ una función que satisface

(i) $f^{\prime}(\infty)=\lambda_{k}$ para algún $k \geq 2$,

(ii) Existe $\gamma>0$ tal que $f^{\prime}(t) \leq \gamma<\lambda_{k+1}$ para todo $t \in \mathbb{R}$,

(iii) $f^{\prime}(0)<\lambda_{1}$.

Sean $\epsilon>0, A>0, D>0$, y $B>0$ como en el Teorema 2.9. Suponga, además, que $f$ satisface

(iv) $F(t)-\frac{1}{2} \lambda_{k} t^{2} \rightarrow \infty$ cuando $|t| \rightarrow \infty$, donde $F(t)=$ $\int_{0}^{t} f(s) d s . S i$

$$
f^{\prime}(t)<\lambda_{k} \quad \forall t \in[-B, B]
$$

entonces existe al menos una solución $u_{*}$ de (10) que cambia de signo y tal que

$$
\left\|\boldsymbol{u}_{*}\right\|_{L^{\infty}(\Omega)}>B
$$


Continuando con el estudio del problema (10), J. Cossio, S. Herrón y C. Vélez, utilizando el teorema de bifurcación global y bifurcación de infinito, demostraron la existencia de múltiples soluciones de (10) cuando la no linealidad es asintóticamente lineal (véase Cossio, Herrón \& Vélez (2013)).

En los siguientes resultados se supone que $\lambda_{2}=\cdots=\lambda_{k}$ es un valor propio de multiplicidad impar y la no linealidad $f$ satisface:

(f $f_{1} f^{\prime}(0):=\lim _{t \rightarrow 0} \frac{f(t)}{t} \in\left(\lambda_{2}, \lambda_{k+1}\right)$,

$\left(f_{2}\right) \quad f^{\prime}(\infty):=\lim _{|t| \rightarrow \infty} \frac{f(t)}{t} \in\left(\lambda_{2}, \lambda_{k+1}\right)$,

$\left(f_{3}\right)$ existe un número positivo $\alpha$ tal que $f(\alpha) \leq 0 \leq$ $f(-\alpha)$.

Teorema 2.13. (Cossio, Herrón \& Vélez (2013)) Si f satisface $\left(f_{1}\right),\left(f_{2}\right)$ y $\left(f_{3}\right)$ entonces el problema (10) tiene al menos seis soluciones no triviales $u_{1}, u_{2}, v_{1}, v_{2}, w_{1} y w_{2}$. Las soluciones $u_{1}$ y $u_{2}$ son positivas en $\Omega$, las soluciones $v_{1}$ y $v_{2}$ son negativas en $\Omega$, y las soluciones $w_{1} y w_{2}$ cambian de signo en $\Omega$. Además,

$$
\left\|u_{1}\right\|_{L^{\infty}, \| v_{1}}\left\|_{L^{\infty}, \| W_{1}}\right\|_{L^{\infty}}<\alpha
$$

y

$$
\left\|u_{2}\right\|_{L^{\infty}, \| v_{2}}\left\|_{L^{\infty}, \| W_{2}}\right\|_{L^{\infty}}>\alpha .
$$

La demostración del Teorema 2.13 utiliza el teorema de bifurcación global y bifurcación de infinito (véanse Rabinowitz (1971), Rabinowitz (1985), Ambrosetti \& Hess (1980), Ambrosetti \& Malchiodi (2007)) aplicados al problema

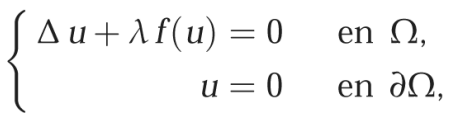

donde $\lambda \in \mathbb{R}$.

Consideremos ahora las siguientes hipótesis más débiles:

$\left(f_{1}^{\prime}\right) f^{\prime}(0)>\lambda_{1}$,

$\left(f_{2}^{\prime}\right) f^{\prime}(\infty)>\lambda_{1}$,

$\left(f_{3}^{\prime}\right)$ existe $\alpha_{-}<0$ y $\alpha_{+}>0$ tal que $f\left(\alpha_{+}\right) \leq 0 \leq f\left(\alpha_{-}\right)$.

Como una consecuencia de la técnica utilizada para demostrar el Teorema 2.13 probamos los siguientes resultados.

Teorema 2.14. (Cossio, Herrón \& Vélez (2013)) Si f satisface

i) $\left(f_{1}^{\prime}\right),\left(f_{2}\right)$ y $\left(f_{3}\right), o$

ii) $\left(f_{1}\right),\left(f_{2}^{\prime}\right)$ y $\left(f_{3}\right)$, entonces (10) tiene al menos cinco soluciones no triviales $u_{1}, u_{2}, v_{1}, v_{2} y w$. Las soluciones $u_{1} y u_{2}$ son positivas en $\Omega$, las soluciones $v_{1}$ y $v_{2}$ son negativas en $\Omega$ y la solución $w$ cambia de signo en $\Omega$. Además,

$$
\left\|u_{1}\right\|_{L^{\infty}}<\alpha<\left\|u_{2}\right\|_{L^{\infty}},
$$

y

$$
\left\|v_{1}\right\|_{L^{\infty}}<\alpha<\left\|v_{2}\right\|_{L^{\infty}} .
$$

Teorema 2.15. (Cossio, Herrón \& Vélez (2013)) Si f satisface $\left(f_{1}^{\prime}\right),\left(f_{2}^{\prime}\right)$ y $\left(f_{3}^{\prime}\right)$ entonces (10) tiene al menos cuatro soluciones $u_{1}, u_{2}, v_{1}, v_{2} \cdot u_{1}$ y $u_{2}$ son positivas en $\Omega$ y $v_{1}$ y $v_{2}$ son negativas en $\Omega$. Además,

$$
\left\|u_{1}\right\|_{L^{\infty}}<\alpha_{+}<\left\|u_{2}\right\|_{L^{\infty}}
$$

y

$$
\left\|v_{1}\right\|_{L^{\infty}}<\left|\alpha_{-}\right|<\left\|v_{2}\right\|_{L^{\infty}} .
$$

Para el resultado siguiente sólo necesitamos suponer que $f$ está definida en un intervalo $[-\alpha, \alpha] \operatorname{con} \alpha>0$.

Teorema 2.16. (Cossio, Herrón \& Vélez (2013)) Sea $f$ : $[-\alpha, \alpha] \longrightarrow \mathbb{R}$ una función Lipschitz que satisface $\left(f_{1}\right)$ y $\left(f_{3}\right)$. Entonces existen $u, v, w \in C^{2}(\bar{\Omega})$ tales que

$$
\|u\|_{L^{\infty},\|v\|_{L^{\infty}},\|W\|_{L^{\infty}}<\alpha,}
$$

y u, v y w son soluciones de (10). Además, u es positiva en $\Omega$, $v$ es negativa en $\Omega$ y $w$ cambia de signo en $\Omega$.

Como una consecuencia del teorema anterior, el problema (10) tiene al menos tres soluciones, con las propiedades mencionadas, si $f: \mathbb{R} \longrightarrow \mathbb{R}$ es Lipschitz, satisface $\left(f_{1}\right),\left(f_{3}\right)$ y tiene un crecimiento arbitario en el infinito.

En los Teoremas 2.13, 2.14, 2.15, 2.16, descritos anteriormente, no necesitamos suponer un acotamiento global de $f^{\prime}$ ni hacemos uso del método de reducción de Lyapunov-Schmidt como en Castro \& Cossio (1994) y Castro, Cossio \& Vélez (2013). En lugar de utilizar métodos variacionales y teoría de grado, utilizamos el teorema de bifurcación global y el teorema de bifurcación de infinito en combinación con el principio del máximo. Como la condición $f^{\prime}(0)<\lambda_{1}$ no es parte de nuestras hipótesis, el funcional de energía asociado a (10) no tiene geometría de tipo paso de montaña alrededor de cero (como si ocurre en Castro \& Cossio (1994) y Castro, Cossio \& Vélez (2013)), y aún así logramos demostrar la existencia de soluciones de un signo. Además, no se supone ninguna clase de simetría ni en $\Omega$ ni en $f$, contrario a muchas referencias que estudian, por ejemplo, el caso radial.

Por otro lado, cuando $\lambda_{2}$ es un valor propio simple y la derivada de la no linealidad está acotada por $\lambda_{3}$, utilizando teoría de grado y el método de reducción de 
Lyapunov-Schmidt, demostramos la existencia de múltiples soluciones y algunas propiedades cualitativas de las soluciones. Para este propósito, usamos el siguiente resultado debido a A. Castro, J. Cossio y J. M. Neuberger (véase Castro, Cossio \& Neuberger (1998)).

Teorema 2.17. (Castro, Cossio \& Neuberger (1998)) Si $f: \mathbb{R} \rightarrow \mathbb{R}$ es una función de clase $C^{2}$ tal que

$$
\begin{aligned}
& \left(l_{1}\right) f^{\prime}(0)<\lambda_{1}, \\
& \left(l_{2}\right) f^{\prime}(\infty) \in\left(\lambda_{2}, \lambda_{3}\right), \\
& \left(l_{3}\right) t f^{\prime \prime}(t)>0,
\end{aligned}
$$

entonces (10) tiene al menos cuatro soluciones no triviales, dos de las cuales cambian de signo.

Bajo las hipótesis del teorema anterior, existe una solución û de (10) tal que

$$
J(\hat{u})=\max _{x \in X}\left(\min _{y \in Y} J(x+y)\right),
$$

donde $X$ es el subespacio de $H_{0}^{1}(\Omega)$ generado por $\left\{\varphi_{1}, \varphi_{2}\right\}, Y=X^{\perp}$ y $J: H_{0}^{1}(\Omega) \rightarrow \mathbb{R}$ está definido por

$$
J(u)=\int_{\Omega}\left(\frac{1}{2}|\nabla u|^{2}-F(u)\right)
$$

$\operatorname{con} F(\xi)=\int_{0}^{\xi} f(s) d s$

Utilizando teoría de grado demostramos el siguiente resultado.

Teorema 2.18. (Cossio, Herrón \& Vélez (2013)) Bajo las mismas hipótesis del Teorema 2.17, si la solución û es positiva (negativa) en $\Omega$ entonces el problema (10) tiene al menos siete soluciones, tres de las cuales tienen el mismo signo.

Bajo hipótesis más fuertes en la no linealidad $f$ podemos dar un número exacto de soluciones de (10).

Teorema 2.19. (Cossio, Herrón \& Vélez (2013)) Supongamos que $\lambda_{2}$ es un valor propio simple. Si $f: \mathbb{R} \rightarrow \mathbb{R}$ es una función de clase $C^{2}$ que satisface $\left(E_{1}\right),\left(E_{2}\right)$ y

$$
\begin{aligned}
& \text { (E) } f^{\prime}(t)<\lambda_{2} \forall t \in[-B, B] \text {, donde } B \text {, }\left(E_{1}\right) \text { y }\left(E_{2}\right) \text { son } \\
& \text { como en el Teorema 2.9, }
\end{aligned}
$$

entonces (10) tiene exactamente cinco soluciones, todas no degeneradas. Además, una es negativa, otra es positiva y existen dos soluciones que cambian de signo exactamente una vez. Las soluciones de un signo tienen índice de Morse 1, las soluciones que cambian de signo tienen índice de Morse 2 y la solución trivial tiene índice de Morse 0.

A continuación establecemos condiciones suficientes para que un problema asintóticamente lineal no resonante tenga al menos siete soluciones. Para ello consideramos el siguiente problema.

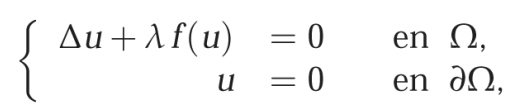

donde $\Omega \subset \mathbb{R}^{N}, N>2$ es un conjunto acotado con frontera suave, $\lambda \in \mathbb{R}$ y $f: \mathbb{R} \rightarrow \mathbb{R}$ es una función de clase $C^{1}$.

Suponemos que $f$ satisface las siguientes propiedades:

(f1) $f^{\prime}(0) \leq 0$

(f2) $\lim _{|t| \rightarrow \infty} f^{\prime}(t)=1$

(f3) $t f^{\prime \prime}(t)>0$ para todo $t \in \mathbb{R}-\{0\}$.

El resultado principal es el siguiente:

Teorema 2.20. (Castro, Cossio \& Vélez (2013)) Si $k \geq 3$ y $\lambda_{k}<\lambda_{k+1}$ entonces existe $\epsilon>0$ tal que si $\lambda \in\left(\lambda_{k+1}, \lambda_{k+1}+\right.$ $\epsilon)$ entonces (24) tiene por lo menos siete soluciones.

En el caso asintóticamente lineal resonante el problema (24) tiene al menos cinco soluciones.

Teorema 2.21. (Castro, Cossio \& Vélez (2013)) Si $\lambda=$ $\lambda_{k+1}$ entonces (24) tiene al menos cinco soluciones.

En las demostraciones de los dos teoremas anteriores se utiliza el teorema del paso de la montaña, argumentos de reducción de Lyapunov-Schmidt, grupos críticos e índices de Morse, existencia de soluciones que cambian de signo exactamente una vez y propiedades de bifurcación.

\section{Problemas elípticos cuasilineales}

En esta sección presentamos algunos resultados que hemos obtenido estudiando la existencia de soluciones para problemas de frontera cuasilineales que involucran el $p$-laplaciano. Como se mencionó en la introducción de este trabajo, es bien conocido que ciertos modelos matemáticos no lineales conducen a ecuaciones diferenciales que contienen el $p$-laplaciano.

En lo que sigue estudiaremos la existencia de soluciones para el problema de frontera cuasilineal

$$
\left\{\begin{aligned}
\Delta_{p} u+f(u)=0 & \text { en } \Omega, \\
u=0 & \text { en } \partial \Omega,
\end{aligned}\right.
$$

donde $\Omega$ es un conjunto abierto acotado en $\mathbb{R}^{N}(N \geq 2)$ y $f: \mathbb{R} \rightarrow \mathbb{R}$ es una función no lineal tal que $f(0)=0$.

Nuestro primer resultado demuestra que (25) tiene múltiples soluciones radiales cuando $\Omega=B_{1}(0) \subset \mathbb{R}^{N}$ 
es la bola unitaria con centro en el origen, la no linealidad $f \in C^{1}(\mathbb{R})$ tiene un cero positivo y el rango de la $p$-derivada de la no linealidad incluye al menos los primeros $j$ valores propios radiales del operador p-laplaciano.

Para este primer resultado suponemos las siguientes hipótesis sobre la no linealidad:

(a) Existen números positivos $\beta_{1}, \beta_{2}$ tales que $f\left(\beta_{1}\right)=$ $0 \mathrm{y}$

$$
\beta_{2}=\inf \left\{t \in\left[\beta_{1}, \infty\right): \forall s>t, f(s)>0\right\} .
$$

(b) Para algún $j \in \mathbb{N}, \lambda_{j}(p)<\lim _{|t| \rightarrow \infty} \frac{f(t)}{|t|^{p-2} t}=: \lambda_{\infty} \in \mathbb{R}$ $\left(\lambda_{j}(p)\right.$ denota el $j$-ésimo valor propio del problema $(8))$.

(c) $\lim _{t \rightarrow 0} \frac{f^{\prime}(t)}{\left(|t|^{p-2} t\right)^{\prime}}=\lambda_{\infty}$.

(d) Existe una constante $C>0$ tal que

$$
\limsup _{t \rightarrow \beta_{i}}\left|\frac{f(t)}{\left|t-\beta_{i}\right|^{p-2}\left(t-\beta_{i}\right)}\right| \leq C \quad \text { para } \quad i=1,2 .
$$

Debido a la hipótesis (b), el problema (25) es $p$ asintóticamente lineal en infinito. El principal resultado es el siguiente:

Teorema 3.1. (Cossio \& Herrón (2008)) Supongamos $p \geq$ 2. Si f satisface las hipótesis (a), (b), (c) y (d) entonces el problema (25) tiene al menos $4 j-1$ soluciones radialmente simétricas.

Este teorema extiende un resultado obtenido por A. Castro y J. Cossio (véase Castro \& Cossio (1993)) para el caso semilineal. Las principales herramientas que utilizamos para demostrar el teorema son un resultado de unicidad para un problema de valor inicial que involucra el $p$-laplaciano, la versión radial para el $p$-laplaciano del teorema global de bifurcación de P. Rabinowitz, debida a M. del Pino y R. Manásevich (véase Del Pino \& Manásevich (1991)). Un resultado del tipo CrandallRabinowitz, debido a J. García Melián y J. Sabina de Lis (véase García-Melián \& Sabina de Lis (2002)) y bifurcación de infinito.

J. Iaia (véase Iaia (1995)) estudió el caso radial del problema

$$
\left\{\begin{aligned}
\Delta_{p} u+|u|^{q-1} u & =0 & & \text { en } \Omega, \\
u & =0 & & \text { en } \partial \Omega,
\end{aligned}\right.
$$

donde $\Omega=\left\{x \in \mathbb{R}^{N}:\|x\|<1\right\}$ es la bola unitaria en $\mathbb{R}^{N}$ y $1<q+1<p<N$. Iaia demostró que (26) tiene infinitas soluciones radialmente simétricas.
En Cossio, Herrón \& Vélez (2011), se extendió el resultado anterior de Iaia a no linealidades más generales, cuando la no linealidad es $p$-superlineal en el origen. La técnica utilizada es el "shooting method" (método del disparo).

Para los siguientes resultados se supone que la no linealidad $f$ es una función continua localmente Lipschitz, que satisface las siguientes condiciones:

(i) Para todo $u \neq 0, u f(u)>0$.

(ii) Existen $\alpha<0, \varrho>0$ y $a>0$ tales que

$$
|u| \leq \varrho \quad \Longrightarrow \quad|f(u)| \geq a|u|^{p-1+\alpha} \text {. }
$$

La condición (ii) y el comportamiento Lipschitziano de $f$ implican que $2-p \leq \alpha$ y que

$$
f_{p}^{\prime}(0)=\lim _{u \rightarrow 0} \frac{f(u)}{|u|^{p-2} u}=\infty,
$$

es decir, el problema es p-superlineal en el origen.

Los resultados obtenidos son:

Teorema 3.2. (Cossio, Herrón \& Vélez (2011)) Supongamos que $p>N$ y $f$ es una función continua localmente Lipschitz que satisface (i), (ii) $y$

(iii) existen $\beta \in(1, p), M>0$ y $b_{1}, b_{2}>0$ tales que

$$
|u|>M \quad \Longrightarrow \quad b_{1}|u|^{p-\beta} \leq|f(u)| \leq b_{2}|u|^{p-\beta} .
$$

Entonces para cada natural $k$, el problema (25) tiene una solución radial con exactamente $k$ ceros interiores en $[0,1]$. En particular el problema (25) tiene infinitas soluciones radiales.

Teorema 3.3. (Cossio, Herrón \& Vélez (2011)) sl Si $p>$ $N$ y $f$ es una función continua localmente Lipschitz que satisface (i) y (ii) entonces existe un entero positivo $k_{0}$ tal que para cada $k \geq k_{0}$ el problema (25) tiene una solución radial con exactamente $k$ ceros interiores en $[0,1]$. En particular el problema (25) tiene infinitas soluciones radiales.

Observamos que resultados como los obtenidos por A. El Hachimi y F. De Thelin (véase El Hachimi \& De Thelin (1996)) y J. Iaia (véase Iaia (1995)) exigen un comportamiento de la no linealidad $f$ en cero y en infinito, mientras que nuestro resultado en el Teorema 3.3 sólo requiere una condición en la no linealidad $f$ en cero. En Bognár \& Drábek (2005), Capietto, Dambrosio \& Zanolin ( 2001), Garcia-Huidrobo, Manásevich \& Schmitt (1999) y O'Regan \& Wang (2008) el lector interesado puede encontrar resultados relacionados con los que hemos obtenido en los teoremas anteriores.

Como se mencionó en el estudio de problemas elípticos semilineales que se presentó en la Sección 2 de este trabajo, J. Cossio, S. Herrón y C. Vélez demostraron (véase Cossio, Herrón \& Vélez (2013)), utilizando teoría de bifurcación, la existencia de seis soluciones para el problema (10) (véase el Teorema 2.13). 
En el siguiente resultado los mismos autores mencionados en el párrafo anterior estudian, en el caso general, el problema

$$
\left\{\begin{aligned}
\Delta_{p} u+f(u)=0 & \text { en } \Omega, \\
u=0 & \text { en } \partial \Omega,
\end{aligned}\right.
$$

donde $\Omega \subset \mathbb{R}^{N}, N \geq 2$, es un dominio acotado con frontera suave, $1<p<2$ y $f: \mathbb{R} \rightarrow \mathbb{R}$ es una función no lineal que satisface:

$\left(f_{1}\right)|f(t)-f(s)| \leq[f]_{0,1}|t-s|^{p-1}, \quad \forall s, t \in \mathbb{R}$,

$\left(f_{2}\right) f_{p}^{\prime}(0)=\lim _{t \rightarrow 0} \frac{f(t)}{|t|^{p-2} t}>\lambda_{1}(p)$,

$\left(f_{3}\right) \quad f_{p}^{\prime}(\infty)=\lim _{|t| \rightarrow \infty} \frac{f(t)}{|t|^{p-2} t}>\lambda_{1}(p)$,

$\left(f_{4}\right)$ existe un número positivo $\alpha$ tal que $f(\alpha) \leq 0 \leq$ $f(-\alpha)$,

donde $[f]_{0,1}:=\sup _{s \neq t}|f(s)-f(t)| /|s-t|^{p-1}$ y $\lambda_{1}(p)$ denota el primer valor propio del problema (8).

Con estas hipótesis se prueba que (27) tiene al menos cuatro soluciones no triviales cuando la $p$-derivada en cero y la $p$-derivada en infinito son mayores que el primer valor propio del p-laplaciano. También se presentan algunas propiedades cualitativas de dichas soluciones.

Teorema 3.4. (Cossio, Herrón \& Vélez (2014)) Si f satisface $\left(f_{1}\right),\left(f_{2}\right),\left(f_{3}\right)$ y $\left(f_{4}\right)$ entonces el problema $(27)$ tiene al menos cuatro soluciones no triviales $u_{1}, u_{2}, v_{1}$, and $v_{2}$. Las soluciones $u_{1}$ y $u_{2}$ son positivas en $\Omega$ y las soluciones $v_{1} y v_{2}$ son negativas en $\Omega$. Además,

$$
\left\|u_{1}\right\|_{L^{\infty}},\left\|v_{1}\right\|_{L^{\infty}}<\alpha
$$

$y$

$$
\left\|u_{2}\right\|_{L^{\infty}},\left\|v_{2}\right\|_{L^{\infty}}>\alpha
$$

La prueba del teorema anterior utiliza el teorema global de bifurcación (véase Del Pino \& Manásevich (1991)), bifurcación de infinito (véase Rabinowitz (1973)) y una versión no lineal del principio del máximo debida a J. L. Vázquez (véase Vázquez (1984)).

\section{Perspectivas}

En esta sección presentamos una serie de preguntas abiertas relacionadas con los problemas elípticos semilineales y cuasilineales, que esperamos esperamos sean de interés para los analistas no lineales y contribuyan a un mejor entendimiento de este tipo de problemas.

\subsection{Problemas elípticos semilineales}

Como se puede observar en la Sección 2 de este trabajo, el autor, en colaboración con A. Castro, S. Herrón y C. Vélez, ha introducido nuevas ideas en el estudio de la existencia, multiplicidad y propiedades cualitativas de las soluciones para el problema elíptico semilineal

$$
\left\{\begin{aligned}
\Delta u+f(u) & =0 & & \text { en } \Omega, \\
u & =0 & & \text { en } \partial \Omega .
\end{aligned}\right.
$$

Estas ideas nos conducen a formular una serie de preguntas abiertas que serán especificadas a continuación.

Perspectiva I. Consideremos el problema

$$
\left\{\begin{aligned}
-\Delta u+W(x) u & =f(u) & & \text { en } \Omega, \\
u & =0 & & \text { en } \partial \Omega .
\end{aligned}\right.
$$

Motivados por los resultados obtenidos en la Sección 2, sería interesante encontrar condiciones sobre el potencial $W$ y sobre la no linealidad $f$ que permitan demostrar la existencia de soluciones no triviales para el problema (29). Una vez encontradas las condiciones de la pregunta anterior sería importante estudiar qué propiedades cualitativas tienen dichas soluciones.

Perspectiva II. Sería interesante demostrar que si se tienen las hipótesis $f^{\prime}(0)<\lambda_{1} y f^{\prime}(\infty) \in\left(\lambda_{k}, \lambda_{k+1}\right)$ entonces el número de soluciones de (28) tiende a infinito cuando $k \rightarrow \infty$.

Perspectiva III. Como el principio de minimax de Castro, Cossio y Neuberger (véase el Teorema 2.6) también vale cuando $f$ se reemplaza por $\lambda f$ en (28), donde $\lambda \in \mathbb{R}$, sería interesante demostrar que las soluciones correspondientes $u_{\lambda}$ están en la misma curva de bifurcación. Conjeturamos que ellas se bifurcan del segundo valor propio y que existe un conjunto conexo de soluciones que cambian de signo exactamente una vez y conecta $\left(\lambda_{2}, \infty\right)$ con $\left(\lambda_{2}, 0\right)$.

Perspectiva IV. Motivados por el caso unidimensional, el caso radial y los resultados del Teorema 2.6, sería importante encontrar condiciones suficientes en la no linealidad $f$ que permitan demostrar que para cada entero positivo $\mathrm{k}$ el problema (28) tiene una solución con $k$ regiones nodales.

\subsection{Problemas elípticos cuasilineales}

En la Sección 3 de este trabajo se presentaron algunos resultados obtenidos por el autor en colaboración con $\mathbf{S}$. Herrón y C. Vélez, en el estudio de la existencia, las propiedades cualitativas y la multiplicidad tanto de las soluciones radiales como de las soluciones generales del problema elíptico cuasilineal

$$
\left\{\begin{aligned}
\Delta_{p} u+f(u) & =0 & & \text { en } \Omega, \\
u & =0 & & \text { en } \partial \Omega,
\end{aligned}\right.
$$


donde $\Omega$ es un conjunto abierto acotado en $\mathbb{R}^{N}, N \geq 2$, y $f: \mathbb{R} \rightarrow \mathbb{R}$ es una función no lineal.

Estos resultados nos conducen a formular las siguientes preguntas:

Perspectiva V. Consideremos el problema

$$
\left\{\begin{aligned}
\Delta_{p} u+f(u) & =0 \quad \text { en } \mathbb{R}^{N}, \\
\lim _{|x| \rightarrow \infty} u(x) & =0 .
\end{aligned}\right.
$$

Motivados por los resultados del Teorema 3.2, sería importante encontrar condiciones en la no linealidad $f$ de tal manera que para cada $n \in \mathbb{N}$ exista una solución radial $u_{n}$ del problema (31) con exactamente $n$ ceros. En otras palabras, encontrar condiciones en la no linealidad $f$ para que el problema (31) tenga infinitas soluciones radiales.

Perspectiva VI. M. del Pino y R. Manásevich estudiaron el problema (30) en el caso en que $\Omega$ es la bola unitaria con centro en el origen $B_{1}\{0\} \subset \mathbb{R}^{N}$ (véase Del Pino \& Manásevich (1991)). Ellos demostraron que el problema (30) tiene $n-k+1$ soluciones radiales no triviales suponiendo que $f$ es una función continua, $f(0)=0$, $f(t) /\left(|t|^{p-2} t\right)$ es acotada y

$$
\lim _{t \rightarrow 0} \frac{f(t)}{\left(|t|^{p-2} t\right)}<\lambda_{k}(p) \leq \lambda_{n}(p)<\liminf _{|t| \rightarrow \infty} \frac{f(t)}{\left(|t|^{p-2} t\right)},
$$

donde $n$ y $k$ son números naturales tales que $k \leq n$.

Sea $f(u)=|u|^{q-1} u+g(u)$ en (30). Motivados por los resultados de $\mathbf{M}$. del Pino y $\mathbf{R}$. Manásevich sería interesante encontrar condiciones en la función $g$ y en el exponente $q$ de tal manera que podamos encontrar el número de soluciones radiales del problema

$$
\left\{\begin{aligned}
\Delta_{p} u+|u|^{q-1} u+g(u) & =0 & & \text { en } B_{1}\{0\} \subset \mathbb{R}^{N}, \\
u(x) & =0 & & \text { en } \partial B_{1}\{0\} .
\end{aligned}\right.
$$

Perspectiva VII. Consideremos el problema de valores propios para el $p$-laplaciano

$$
\left\{\begin{aligned}
\Delta_{p} u+\lambda|u|^{p-2} u & =0 & & \text { en } \Omega, \\
u & =0 & & \text { en } \partial \Omega .
\end{aligned}\right.
$$

Siguiendo los lineamientos de P. Drábek (véase Drábek (2007)) planteamos las siguientes preguntas:

(a) ¿Existen valores propios no variacionales de (33)?

(b) ¿Si existen valores propios no variacionales, cuántos hay? ¿Existe un continuo de valores propios no variacionales?

(c) ¿Es el segundo valor propio $\lambda_{2}$ aislado? ¿Existe una sucesión de valores propios no variacionales $\left\{\lambda_{k}\right\}$ de (33) tales que $\lambda_{k} \rightarrow \lambda_{2}$ ?

\section{Referencias}

Aftalion, A., Pacella, F. (2004). Qualitative properties of nodal solutions of semilinear elliptic equations in radially symmetric domains. C. R. Acad. Sci. Paris 339(5): 339-344.

Ambrosetti, A., Hess, P. (1980). Positive solutions of asymptotically linear elliptic eigenvalue problems. J. Math. Anal. Appl.73(2): 411-422.

Ambrosetti, A., Malchiodi, A. (2007). Nonlinear Analysis and Semilinear Elliptic Problems New York: Cambridge Studies in Advanced Mathematics 104, Cambridge University Press.

Ambrosetti, A., Prodi, G. (1993). A Primer of Nonlinear Analysis New York: Cambridge Studies in Advanced Mathematics 34, Cambridge University Press.

Ambrosetti, A., Rabinowitz, P. (1973). Positive solutions of asymptotically linear elliptic eigenvalue problems. J. Math. Anal. 14(2): 349-381.

Anane, A. (1987). Simplicité et isolation de la première valeur prope du p-Laplacien avec poids. Comptes Rendus Acad. Sc. Paris. 305 (16):725-728.

Anane, A., Tsouli, N. (1996). A Primer of Nonlinear Analysis In Nonlinear partial differential equations, Pitman Res. Notes Math., 343, 1-9.

Bartsch,T., Wang. Z, Q. (1996). On the Existence of sign changing solutions for semilinear Dirichlet problems. Topol. Methods Nonlinear Anal. 7: 115-131.

Bartsch, T., Chang, K. C., Wang, Z. Q. (2000). On the Morse indices of sign changing solutions of nonlinear elliptic problems. Math. Z. 233: 655-677.

Bartsch,T., Weth, R. (2003). A note on additional properties of sign-changing solutions to superlinear elliptic equations. Topol. Methods Nonlinear Anal. 22 (1); 1-14.

Bartsch,T., Weth, R., Willem, M. (2005). Partial symmetry of least energy nodal solutions to some variational problems. $J$. Anal. Math. 96: 1-18.

Bognár, G., Drábek, P. (2005). The $p$-Laplacian equation with superlinear and supercritical growth, multiplicity of radial solutions. Nonlinear Analysis 60 (4): 710-728.

Capietto, A., Dambrosio, W., Zanolin, F. (2001). Infinitely many radial solutions to a boundary value problem in a ball. Annali di Matematica Pura ed Applicata 179 (1): 159-188.

Castro, A. (1981). Métodos de Reducción via Minimax Medellín. Primer Simposio Colombiano de Análisis Funcional.

Castro, A., Cossio, J. (1994). Multiple solutions for a nonlinear Dirichlet problem. SIAM J. Math. Anal. 25 1554-1561.

Castro, A., Cossio, J. (1993) Multiple solutions for a nonlinear Dirichlet problem. Rev. Col. Mat. 27(1-2). 15-24. 
Castro, A., Cossio, J. (2006) Construction of a radial solution to a superlinear Dirichlet problem that changes sign exactly once ple solutions for a nonlinear Dirichlet problem En: Basel: Birkhäuse. Contributions to nonlinear analysis. Progr. Nonlinear Differential Equations Appl., 66, 149-160.

Castro, A., Cossio, J. (1993). Multiple solutions for a nonlinear Dirichlet problem. Rev. Col. Mat. 27(1-2): 15-24.

Castro, A., Cossio, J. (2006). Construction of a radial solution to a superlinear Dirichlet problem that changes sign exactly once En: Basel: Birkhäuse. Contributions to nonlinear analysis. Progr. Nonlinear Differential Equations Appl. 66, 149-160.

Castro, A., Cossio, J., Neuberger, J. M. (1997). A sign changing solution for a superlinear Dirichlet problem. Rocky Mountain J. M. 27: 1041-1053.

Castro, A., Cossio, J., Neuberger, J. M. (1997). On Multiple Solutions of a Nonlinear Dirichlet Problem. Nonlinear Analysis, Theory, Methods \& Applications 30 (6): 3657-3662.

Castro, A., Cossio, J., Neuberger, J. M. (1998). A Minmax Principle, Index of the Critical Point, and Existence of Signchanging Solutions to Elliptic Boundary Value Problems. Electronic Journal of Differential Equations 30: 1-18.

Castro, A., Cossio, J., Vélez, C. (2013). Existence and qualitative properties of solutions for nonlinear Dirichlet problems. Discrete Contin. Dyn. Sys. 35 (1): 123-140.

Castro, A., Cossio, J., Vélez, C. (2013A). Existence of seven solutions for an asymptotically linear Dirichlet problem without symmetries. Ann. Mat. Pura Appl. 192 (4): 607-619.

Castro, A., Lazer, A. (1976). Applications of a Max-min Principle. Rev. Col. Mat. 10: 141-149.

Castro, A., Lazer, A. (1979). Critical Point Theory and the Number of Solutions of a Nonlinear Dirichlet Problem. Ann. Mat. Pura Appl. 70 (4): 113-137.

Chang, K. C. (1981). Solutions of asymptotically linear operator equations via Morse theory. Comm. Pure Appl. Math 34 (5): 693-712.

Chang, K. C. (1993). Infinite dimensional Morse theory and multiple Solution Problems. Boston: Birkhäuser.

Chang, K. C., Li, S., Liu, J. (1994). Remarks on multiple solutions for asymptotically linear elliptic boundary value problems. Topol. Methods in Nonlinear Anal. 3: 179-187.

Cossio, J. (1996). Múltiples soluciones para un problema elíptico semilineal. En: Memorias Tercera Escuela de Verano en Geometría diferencial, Ecuaciones diferenciales y Análisis numérico. Memoria No. 7. Bogotá, Academia Colombiana de Ciencias Exactas, Fśicas y Naturales: 53-597.

Cossio, J. (2004). Contribución al estudio de las ecuaciones diferenciales parciales de tipo elíptico. Rev. Acad. Colomb. Cienc. 28 (106): 135-145.
Cossio, J., Herrón, J. (2004). Nontrivial solutions for a semilinear Dirichlet problem with nonlinearity crossing multiples eigenvalues. J. Dynam. Differential Equations. 16 (3): 795-803.

Cossio, J., Herrón, J. (2008). Existence of radial solutions for an asymptotically linear $p$-Laplacian problem. J. Math. Anal. Appl. 345 (1): 583-592.

Cossio, J., Herrón, J., Vélez, C. (2011). Infinitely many radial solutions for a $p$-Laplacian problem $p$-superlinear at the origin. J. Math. Anal. Appl. 376 (2): 741-749.

Cossio, J., Herrón, J., Vélez, C. (2009). Existence of solutions for an asymptotically linear Dirichlet problem via LazerSolimini results. Nonlinear Anal. 71 (1-2:, 66-71.

Cossio, J., Herrón, J., Vélez, C. (2013). Multiple solutions for nonlinear Dirichlet problems via bifurcation and additional results. J. Math. Anal. Appl. 399 (1): 166-179.

Cossio, J., Herrón, J., Vélez, C. (2014). Existence of solutions for a quasilinear elliptic problem. Preprint.

Cossio, J., Vélez, C. (2003). Nontrivial solutions of an asymtotically linear Dirichlet problem. Rev. Col. Mat. 37 (1): 25-36.

Crandall, M., Rabinowitz, P. (1971). Bifurcation from simple eigenvalues. J. Funct. Anal. 8 (2): 321-340.

Dancer, E. (1976). Counterexamples to Some Conjectures on the Number of Solutions of Nonlinear Equations. Math. Ann. 16: 1361-1376.

Del Pino, M., Manásevich, R. (1991). Global bifurcation from the eigenvalues of the $p$-Laplacian. Journal of Diff. Eqns. 92: 226-251.

Drábek, P. (2007). The p-Laplacian-Mascot of nonlinear analysis. Acta Math. Univ. Comenianae. LXXVI (1): 85-98.

Drábek, P., Robinson, S. (1999). Resonance problems for the p-Laplacian. J. Functional Analysis. 169: 189-200.

Garcia-Huidrobo, M., Manásevich, R., Schmitt, K. (1999). Positive radial solutions of quasilinear elliptic partial differential equations on a ball. Nonlinear Anal. 35 (2): 175-190.

García-Melián, J., Sabina de Lis, J. (2002). A local bifurcation theorem for degenerate elliptic equations with radial. $J$. Differential equations. 179: 27-43.

Genoud, F. (2011). Bifurcation from infinity for an asymptotically linear problem on the half-line. Nonlinear Anal. 74 (13): 4533-4543.

El Hachimi, A. De Thelin, A. (1996). Infinitely Many Radially Symmetric Solutions for a Quasilinear Elliptic Problem in a Ball. Journal of Differential Equations. 128: 78-102.

Iaia, J. (1995). Radial Solutions to a p-Laplacian Dirichlet Problem. Applicable Analysis. 58: 335-350.

Landesman, E. M., Lazer, A. C., Meyers, D. (1975). On Saddle Point Problems in the Calculus of Variations, the Ritz Algorithm, and Monotone Convergence. J. Math. Anal. Appl. 53: 94-614. 
Lazer, A. C., Solimini, S. (1988). Nontrivial Solutions of Operator Equations and Morse Indices of Critical Points of MinMax Type. Nonlinear Anal. 12 (8), 761-775.

Liu, S. (2007). Nontrivial Remarks on multiple solutions for elliptic resonant problems. J. Math. Anal. Appl. 336: 498-505.

Ma, R. Xu, J. Han, X. (2011). Global bifurcation of positive solutions of a second-order periodic boundary value problem with indefinite weight. Nonlinear Anal. 74 (10): 3379-3385.

Morse, M. (1925). Relations between the Critical Points of a Real Function of $n$ Independent Variables. Trans. Amer. Math. Soc. 27: 345-396.

Morse, M. (1934). The Calculus of Variations in the Large. Amer. Math. Soc. Colloq. Publ. 18.

O'Regan, D., Wang, H (2008). Positive radial solutions for p-Laplacian systems. Aequationes Math. 75: 43-50.

Palais, R., Smale, S. (1964). A Generalized Morse Theory. Bull. Amer. Math. Soc. 70: 165-171.

Rabinowitz, P. H. (1971). Some Global Results for Nonlinear Eigenvalue Problems. J. Funct. Anal. 7: 487-513.
Rabinowitz, P. H. (1973). Some aspects of nonlinear eigenvalue problems. Rocky Mountain J. Math. 3: 161-202.

Rabinowitz, P. H. (1985). Topological Methods in Bifurcation Theory. Montreal: Séminaire de Mathématiques Supérieures, Séminaire Scientifique OTAN, Les presses de l'Université de Montreal.

Rabinowitz, P. H. (1986). Minimax Methods in Critical Point Theory with Applications to Differential equations. Providence R.I.: AMS, Regional Conference Series in Mathematics, 65.

Rabinowitz, P. H., Su, J., Wang, Z. Q. (2007). Multiple solutions of superlinear elliptic equations. Atti Accad. Naz. Lincei Cl. Sci. Fis. Mat. Natur. Rend. Lincei. 18 (1): 97-108.

Vázquez, J. L. (1984). A strong maximum principle for some quasilinear elliptic equations. Appl. Math. Optim. 12: 191202.

Wang, Z. Q. (1991). On a superlinear elliptic equation. Ann. Inst. H. Poincaré. Analyse Non Lineaire. 8: 43-57.

Yanagida, E. (1996). Structure of radial solutions to $\Delta u+$ $K(|x|)|u|^{p-1} u=0$ en $\mathbb{R}^{N}$. SIAM J. Math. Anal. 27: 997-1014. 